Publ. Mat. 65 (2021), 389-407

DOI: 10.5565 /PUBLMAT6512114

\title{
RELATING SECOND ORDER GEOMETRY OF MANIFOLDS THROUGH PROJECTIONS AND NORMAL SECTIONS
}

\author{
P. Benedini Riul and R. Oset Sinha
}

\begin{abstract}
We use normal sections to relate the curvature locus of regular (resp. singular corank 1) 3-manifolds in $\mathbb{R}^{6}$ (resp. $\mathbb{R}^{5}$ ) with regular (resp. singular corank 1) surfaces in $\mathbb{R}^{5}$ (resp. $\mathbb{R}^{4}$ ). For example, we show how to generate a Roman surface by a family of ellipses different to Steiner's way. We also study the relations between the regular and singular cases through projections. We show that there is a commutative diagram of projections and normal sections which relates the curvature loci of the different types of manifolds, and therefore, that the second order geometry of all of them is related. In particular, we define asymptotic directions for singular corank 1 3 -manifolds in $\mathbb{R}^{5}$ and relate them to asymptotic directions of regular 3-manifolds in $\mathbb{R}^{6}$ and singular corank 1 surfaces in $\mathbb{R}^{4}$.
\end{abstract}

2010 Mathematics Subject Classification: Primary: 57R45; Secondary: 53A05, $58 \mathrm{~K} 05$.

Key words: projections, normal sections, curvature locus, immersed surfaces, immersed 3-manifolds, singular corank 1 manifolds.

\section{Introduction}

The study of second order geometry of manifolds in Euclidean spaces dates as far back as Gauss. By second order geometry we refer to any geometrical aspects which can be captured by the second fundamental form or, in modern terminology, by the 2-jet of a parametrisation of the manifold. Concepts such as elliptic/parabolic/hyperbolic points, normal curvature, asymptotic directions, and some aspects of the contacts with hyperplanes and spheres are included in the study of second order geometry.

In his seminal paper [15], Little studied second order geometry of immersed manifolds in Euclidean spaces of dimensions greater than 3. In particular, special attention was given to immersed surfaces in $\mathbb{R}^{4}$.

P. Benedini Riul was supported by FAPESP Grant 2019/00194-6. R. Oset Sinha was partially supported by MICINN Grant PGC2018-094889-B-I00 and GVA Grant $\mathrm{AICO} / 2019 / 024$. 
Little defined the second fundamental form and the curvature locus, which is an ellipse in this case. The curvature locus is the image in the normal space by the second fundamental form of the unitary tangent vectors. It can also be seen as the curvature vectors of normal hyperplane sections of the surface. The curvature ellipse is not an affine invariant but its topological type and its position with respect to the origin is an affine invariant. Besides, all the second order geometry is captured by this object.

The introduction of Singularity Theory techniques to study the differential geometry of manifolds in Euclidean spaces has given a great impulse to this subject in the last twenty years. There are many papers devoted to regular surfaces in $\mathbb{R}^{4}$ such as $[\mathbf{9}, \mathbf{1 0}, \mathbf{1 3}, \mathbf{1 7}, \mathbf{1 8}, \mathbf{2 2}, \mathbf{2 3}, \mathbf{2 4}]$, amongst others. For surfaces in $\mathbb{R}^{5},[\mathbf{1 1}, \mathbf{2 0}, \mathbf{2 5}]$ are good examples. In fact, there is a recent book which covers these topics $([\mathbf{1 4}])$. The study of regular 3 -manifolds in $\mathbb{R}^{6}$ is also very recent. Here, the curvature locus is a Veronese surface with many different topological types (see $[\mathbf{7}, \mathbf{8}]$ ).

The interest, however, both for singularists and differential geometers has turned to the study of singular manifolds ([26]). In this paper we deal with manifolds in Euclidean spaces with corank 1 singularities, i.e. the corank of the differential of the parametrisation at any point is at most 1 . For singular corank 1 surfaces in $\mathbb{R}^{N}, N=3,4$, we can cite $[\mathbf{3}, \mathbf{4}, \mathbf{5}, \mathbf{1 6}]$, and for singular corank 13 -manifolds in $\mathbb{R}^{5},[\mathbf{6}]$. Here, the curvature locus is a parabola or a parabolic version of a Veronese surface. Curvature loci in general have been studied in [21], for example.

Our results are mostly local, so we consider germs of manifolds at a point $p$, that is, a $k$-manifold in $\mathbb{R}^{N}$ is parametrised by $f:\left(\mathbb{R}^{k}, 0\right) \rightarrow$ $\left(\mathbb{R}^{N}, p\right)$.

The aim of this paper is to relate the geometry of all these objects which have traditionally been studied separately. There is a natural relationship between regular and singular objects. When projecting orthogonally along a tangent direction a regular $k$-manifold in $\mathbb{R}^{N}$ one obtains a singular $k$-manifold in $\mathbb{R}^{N-1}$. On the other hand, taking normal hyperplane sections of the $k$-manifold gives a family of $(k-1)$-manifolds in one dimension less. In Section 4 we establish a commutative diagram using projections and normal sections which induces a commutative diagram amongst the curvature loci with immersions and blow-ups. As a result of this we prove our main result, that the second order geometry of all these objects is related. This justifies known relations for projections when $k=2$ and $N=4$, for example, and motivates to look for further relations between the geometries of different manifolds, both regular and singular, in different Euclidean spaces. 
Section 2 is devoted to preliminary results on the geometry of all the different objects appearing throughout the paper. In Section 3 we study normal sections of 3-manifolds, both for the regular and singular cases, and show that the curvature locus of a 3-manifold can be generated by the curvature loci of the surfaces obtained by normal sections. In particular, we show how a Roman Steiner surface or a cross-cap surface can be generated by ellipses. In [2] it is shown how using these normal sections some geometry of the 3-manifold can be recovered by the topological types of the curvature loci of the sections.

In Section 5, inspired by the commutative diagram of Section 4, we define asymptotic directions for singular 3-manifolds in $\mathbb{R}^{5}$ and relate them to asymptotic directions of regular 3 -manifolds in $\mathbb{R}^{6}$ and singular surfaces in $\mathbb{R}^{4}$. We prove that if the direction of projection is asymptotic, then it becomes a null tangent direction in the singular projection and so justify the existence of infinite asymptotic directions in the singular case, which was not fully understood until now.

Acknowledgements. The authors would like to thank M. A. S. Ruas for useful conversations and constant encouragement and the referees for a careful reading of the manuscript and invaluable suggestions.

\section{The geometry of regular and singular corank 1 manifolds in Euclidean spaces}

Given a smooth $k$-dimensional manifold $M_{\text {reg }}^{k} \subset \mathbb{R}^{N}, N>k$, and $f: U \rightarrow \mathbb{R}^{N}$ a local parametrisation of $M_{\text {reg }}^{k}$ with $U \subset \mathbb{R}^{k}$ an open subset, let $\left\{e_{1}, \ldots, e_{N}\right\}$ be an orthonormal frame of $\mathbb{R}^{N}$ such that at any $u \in U$, $\left\{e_{1}(u), \ldots, e_{k}(u)\right\}$ is a basis for $T_{p} M_{\mathrm{reg}}^{k}$ and $\left\{e_{k+1}(u), \ldots, e_{N}(u)\right\}$ is a basis for $N_{p} M_{\text {reg }}^{k}$ at $p=f(u)$.

The second fundamental form of $M_{\text {reg }}^{k}$ at a point $p$ is a symmetric bilinear map $I I_{p}: T_{p} M_{\text {reg }}^{k} \times T_{p} M_{\text {reg }}^{k} \rightarrow N_{p} M_{\text {reg }}^{k}$, given by $I I_{p}(v, w)=$ $\pi_{2}\left(d^{2} f(v, w)\right)$, where $\pi_{2}: T_{p} \mathbb{R}^{N} \rightarrow N_{p} M_{\text {reg }}^{k}$ is the canonical projection.

Furthermore, the second fundamental form of $M_{\text {reg }}^{k}$ at $p$ along a normal vector field $\nu$ is the bilinear map $I I_{p}^{\nu}: T_{p} M_{\text {reg }}^{k} \times T_{p} M_{\text {reg }}^{k} \rightarrow \mathbb{R}$, given by $I I_{p}^{\nu}(v, w)=\left\langle\nu, I I_{p}(v, w)\right\rangle$.

For singular $k$-dimensional manifolds with corank 1 singularities, we shall need the following construction, that can be found in $[\mathbf{1 6}]$. A singular manifold $M_{\text {sing }}^{k} \subset \mathbb{R}^{N}$ at $p$ is given by the image of a smooth map $g: \tilde{M} \rightarrow \mathbb{R}^{N}$, where $\tilde{M}$ is a smooth regular $k$-dimensional manifold and $q \in \tilde{M}$ is a corank 1 point of $g$ such that $g(q)=p$. Also, 
consider $\phi: U \rightarrow \mathbb{R}^{k}$ a local coordinate system defined in an open neighbourhood $U$ of $q$ at $\tilde{M}$. Hence, we may consider a local parametrisation $f=g \circ \phi^{-1}$ of $M_{\text {sing }}^{k}$ at $p$ (see the diagram below).

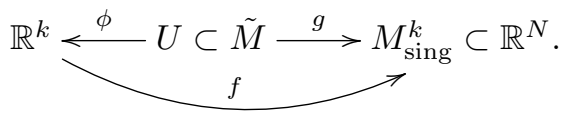

The $(k-1)$-dimensional tangent space of $M_{\text {sing }}^{k}$ at $p, T_{p} M_{\text {sing }}^{k}$, is given by $\operatorname{Im} d g_{q}$, where $d g_{q}: T_{q} \tilde{M} \rightarrow T_{p} \mathbb{R}^{N}$ is the differential map of $g$ at $q$. Thus, the $(N-k+1)$-dimensional normal space of $M_{\text {sing }}^{k}$ at $p, N_{p} M_{\text {sing }}^{k}$, is the subspace orthogonal to $T_{p} M_{\text {sing }}^{k}$ satisfying $T_{p} M_{\text {sing }}^{k} \oplus N_{p} M_{\text {sing }}^{k}=$ $T_{p} \mathbb{R}^{N}$.

The first fundamental form of $M_{\text {sing }}^{k}$ at $p, I: T_{q} \tilde{M} \times T_{q} \tilde{M} \rightarrow \mathbb{R}$, is given by

$$
I(u, v)=\left\langle d g_{q}(u), d g_{q}(v)\right\rangle \quad \forall u, v \in T_{q} \tilde{M} .
$$

Since $g$ has corank 1 at $q$, one can find $0 \neq u \in T_{q} \tilde{M}$ such that $d g_{q}(u)=0$ and $I(u, u)=0$. Therefore the first fundamental form is not a Riemannian metric on $T_{q} \tilde{M}$, but a pseudometric instead. Let $\left(x_{1}, \ldots, x_{k}\right)$ be the Cartesian coordinate system in $\mathbb{R}^{k}$. Taking the frame $\mathcal{B}=\left\{\partial x_{1}, \ldots\right.$, $\left.\partial x_{k}\right\}$ of $T_{q} \tilde{M}$, the coefficients of the first fundamental form of $M_{\text {sing }}^{k}$ at $p$ with respect to $\phi$ are given by $E_{x_{i} x_{j}}(q)=I\left(\partial_{x_{i}}, \partial_{x_{j}}\right)=\left\langle f_{x_{i}}, f_{x_{j}}\right\rangle(\phi(q))$, $1 \leq i, j \leq k$, where $f_{x_{i}}=\frac{\partial f}{\partial x_{i}}$.

Following [16], consider the orthogonal projection $\pi_{2}: T_{p} \mathbb{R}^{N} \rightarrow N_{p} M_{\text {sing }}^{k}$. The second fundamental form of $M_{\text {sing }}^{k}$ at $p, I I: T_{q} \tilde{M} \times T_{q} \tilde{M} \rightarrow N_{p} M_{\text {sing }}^{k}$, in the basis $\mathcal{B}$ of $T_{q} \tilde{M}$, is given by $I I\left(\partial_{x_{i}}, \partial_{x_{j}}\right)=\pi_{2}\left(f_{x_{i} x_{j}}(\phi(q))\right), 1 \leq$ $i, j \leq k$, and we extend it to the whole space in a unique way as a symmetric bilinear map. Given a normal vector $\nu \in N_{p} M_{\text {sing }}^{k}$, we define the second fundamental form along $\nu, I I_{\nu}: T_{q} \tilde{M} \times T_{q} \tilde{M} \rightarrow \mathbb{R}$, given by $I I_{\nu}(u, v)=\langle I I(u, v), \nu\rangle$ for all $u, v \in T_{q} \tilde{M}$.

2.1. The curvature loci. Given a $k$-dimensional manifold $M^{k} \subset \mathbb{R}^{N}$, $N>k$, the curvature locus at a point $p \in M^{k}$ is the set $\left\{I I_{p}(u, u)\right.$ : $\left.u \in T_{p} M^{k}, I(u, u)^{\frac{1}{2}}=1\right\} \subset N_{p} M^{k}$. The curvature locus of a manifold contains all its second order geometry. Any isometric scalar invariant of the curvature locus is an isometric scalar invariant of the manifold, since rotations in $T_{p} M^{k}$ leave invariant the locus and rotations in $N_{p} M^{k}$ rotate the locus. The contact geometry of the manifold is affine invariant. The curvature locus is not affine invariant, but the position with respect to the origin and, in some cases, the topological type are affine invariant. 
Regular case. For a regular manifold $M_{\mathrm{reg}}^{k} \subset \mathbb{R}^{N}$, the curvature locus is also the image of the map $\eta: \mathbb{S}^{k-1} \subset T_{p} M_{\text {reg }}^{k} \rightarrow N_{p} M_{\text {reg }}^{k}$, where $\eta(u)=$ $I I_{p}(u, u)$.

When $k=2$, the curvature locus is an ellipse denoted by $\Delta_{e}$ that may degenerate into a segment or a point. Taking $u=(\cos \theta) e_{1}+(\sin \theta) e_{2} \in$ $\mathbb{S}^{1}, \Delta_{e}$ can be parametrised by

$$
\eta(\theta)=\sum_{i=1}^{N-2}\left(l_{i} \cos ^{2} \theta+2 m_{i} \cos \theta \sin \theta+n_{i} \sin ^{2} \theta\right) e_{2+i},
$$

where $l_{i}=\left\langle f_{x x}, e_{2+i}\right\rangle, m_{i}=\left\langle f_{x y}, e_{2+i}\right\rangle$, and $n_{i}=\left\langle f_{y y}, e_{2+i}\right\rangle, i=1, \ldots, N-$ 2 , are the coefficients of the second fundamental form, and $(x, y)$ are the local coordinates of $\mathbb{R}^{2}$.

For the case where $k=3$ and $N=6$, the curvature locus is denoted by $\Delta_{v}$. The authors show in [7] that taking spherical coordinates in $\mathbb{S}^{2} \subset$ $T_{p} M_{\text {reg }}^{3}$, one can parametrise the curvature locus of $M_{\text {reg }}^{3}$ at $p$ by $\eta: \mathbb{S}^{2} \subset$ $T_{p} M_{\mathrm{reg}}^{3} \rightarrow N_{p} M_{\mathrm{reg}}^{3},(\theta, \phi) \mapsto \eta(\theta, \phi)$, where

$$
\begin{aligned}
\eta(\theta, \phi)= & H+(1+3 \cos (2 \phi)) B_{1}+\cos (2 \theta) \sin ^{2} \theta B_{2} \\
& +\sin (2 \theta) \sin ^{2} \phi B_{3}+\cos \theta \sin (2 \phi) B_{4}+\sin \theta \sin (2 \phi) B_{5}
\end{aligned}
$$

with

$$
\begin{gathered}
H=\frac{1}{3}\left(f_{x x}+f_{y y}+f_{z z}\right), \quad B_{1}=\frac{1}{12}\left(-f_{x x}-f_{y y}+2 f_{z z}\right), \\
B_{2}=\frac{1}{2}\left(f_{x x}-f_{y y}\right), \quad B_{3}=f_{x y}, \quad B_{4}=f_{x z}, \quad B_{5}=f_{y z} .
\end{gathered}
$$

The first normal space is $N_{p}^{1} M_{\text {reg }}^{3}=\left\langle H, B_{1}, B_{2}, B_{3}, B_{4}, B_{5}\right\rangle_{(p)}$. The affine hull of the curvature locus is denoted by $\operatorname{Aff}_{p}$ and the linear subspace of $N_{p}^{1} M_{\text {reg }}^{3}$ parallel to $\operatorname{Aff}_{p}$ by $E_{p}$. The curvature locus of a regular 3 -manifold in $\mathbb{R}^{N}$ can be seen as the image of the classical Veronese surface of order 2 via a convenient linear map.

In $[\boldsymbol{7}]$ it is shown that the curvature locus at a point $p$ where $\operatorname{dim}\left(N_{p}^{1} M_{\text {reg }}^{3}\right)=3$ in a 3 -manifold $M_{\text {reg }}^{3} \subset \mathbb{R}^{6}$ has one of the following shapes: a Roman Steiner surface (Figure 1), a cross-cap surface (Figure 2), a Steiner surface of type 5 (Figure 3), a cross-cup surface (Figure 4), an ellipsoid, a (compact) cone, or a planar region. 


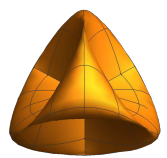

FiguRE 1. Roman Steiner surface.

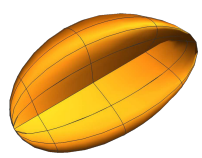

Figure 3. Steiner surface of type 5.

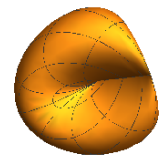

Figure 2. Cross-cap surface.

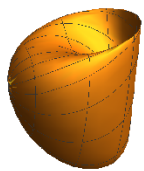

Figure 4. Cross-cup surface.

Singular case. The curvature locus at a singular corank 1 point $p$ of a $k$-dimensional manifold $M_{\text {sing }}^{k} \subset \mathbb{R}^{N}, N>k$, is also given by the image of the map $\eta: C_{q} \rightarrow N_{p} M$ defined by $\eta(u)=I I(u, u)$, where $C_{q} \subset T_{q} \tilde{M}$ is the subset of unit tangent vectors (i.e. vectors $u \in T_{q} \tilde{M}$ such that $\left.I(u, u)^{\frac{1}{2}}=1\right)$.

When $k=2$, the curvature locus denoted by $\Delta_{p}$ is a parabola that may degenerate into a half-line, a line, or a point. In the same way as the curvature ellipse does for regular surfaces, it contains information about the second order geometry of the surface. For $N=3$, the normal space is a plane. However, if $N>3$, the normal space has higher dimension and the plane that contains $\Delta_{p}$ must be defined, specially when $\Delta_{p}$ is degenerate. The minimal affine space that contains the curvature parabola is denoted by $\mathrm{Aff}_{p}$. The plane denoted by $E_{p}$ is the vector space: parallel to $\operatorname{Aff}_{p}$ when $\Delta_{p}$ is a non degenerate parabola, the plane through $p$ that contains $\operatorname{Aff}_{p}$ when $\Delta_{p}$ is a non radial half-line or a non radial line, and any plane through $p$ that contains $\operatorname{Aff}_{p}$ when $\Delta_{p}$ is a radial half-line, a radial line, or a point.

When $k=3$, the curvature locus is denoted by $\Delta_{c v}$. Some examples of topological types of $\Delta_{c v}$ can be found in [6].

It is possible to take a coordinate system $\phi$ and make rotations in the target in order to obtain a local parametrisation for $M_{\text {sing }}^{k}$ at $p$ given by

$$
f\left(x_{1}, \ldots, x_{k}\right)=\left(x_{1}, \ldots, x_{k-1}, f_{k}\left(x_{1}, \ldots, x_{k}\right), \ldots, f_{N}\left(x_{1}, \ldots, x_{k}\right)\right),
$$

where $\frac{\partial f_{i}}{\partial x_{j}}(\phi(q))=0$ for $i=k, \ldots, N$ and $1 \leq j \leq k$. Hence, the subset of unit tangent vectors $C_{q} \in T_{q} \tilde{M}$ is the cylinder given by $\left\{\left(a_{1}, \ldots, a_{k}\right) \in\right.$ $\left.T_{q} \tilde{M}: a_{1}^{2}+\cdots+a_{k-1}^{2}=1\right\}$. Taking an orthonormal frame $\left\{\nu_{1}, \ldots, \nu_{N-k+1}\right\}$ of $N_{p} M_{\text {sing }}^{k}$, the curvature locus $\Delta_{p}$ can be parametrised by: 
(i) If $k=2, C_{q}=\left\{(a, b) \in T_{q} \tilde{M}: a^{2}=1\right\}$, and the parametrisation is

$$
\eta(b)=\sum_{i=1}^{N-1}\left(l_{\nu_{i}}+2 m_{\nu_{i}} b+n_{\nu_{i}} b^{2}\right) \nu_{i}
$$

where $l_{\nu_{i}}(q)=\left\langle\pi_{2}\left(f_{x x}\right), \nu_{i}\right\rangle(\phi(q)), m_{\nu_{i}}(q)=\left\langle\pi_{2}\left(f_{x y}\right), \nu_{i}\right\rangle(\phi(q))$, and $n_{\nu_{i}}(q)=\left\langle\pi_{2}\left(f_{y y}\right), \nu_{i}\right\rangle(\phi(q)), 1 \leq i \leq N-1$, are the coefficients of the second fundamental form, $(x, y)$ are local coordinates of $\mathbb{R}^{2}$, and the parameter $b \in \mathbb{R}$ corresponds to a unit tangent direction $u=$ $\partial_{x}+b \partial_{y}=(1, b) \in C_{q}$.

(ii) If $k=3, C_{q}=\left\{(a, b, c) \in T_{q} \tilde{M}: a^{2}+b^{2}=1\right\}$, and the parametrisation is

$$
\eta(a, b, c)=\sum_{i=1}^{N-2}\left(a^{2} l_{\nu_{i}}+2 a b m_{\nu_{i}}+b^{2} n_{\nu_{i}}+c^{2} p_{\nu_{i}}+2 a c q_{\nu_{i}}+2 b c r_{\nu_{i}}\right) \nu_{i}
$$

where $a^{2}+b^{2}=1$,

$$
\begin{aligned}
l_{\nu}(q) & =\left\langle\pi_{2}\left(f_{x x}\right), \nu\right\rangle, \quad m_{\nu}(q)=\left\langle\pi_{2}\left(f_{x y}\right), \nu\right\rangle, \quad n_{\nu}(q)=\left\langle\pi_{2}\left(f_{y y}\right), \nu\right\rangle, \\
p_{\nu}(q) & =\left\langle\pi_{2}\left(f_{z z}\right), \nu\right\rangle, \quad q_{\nu}(q)=\left\langle\pi_{2}\left(f_{x z}\right), \nu\right\rangle, \quad r_{\nu}(q)=\left\langle\pi_{2}\left(f_{y z}\right), \nu\right\rangle,
\end{aligned}
$$

are the coefficients of the second fundamental form with all the partial derivatives evaluated at $\phi(q)$, and $(x, y, z)$ are local coordinates of $\mathbb{R}^{3}$.

For singular corank 1 surfaces, $k=2$, a non zero direction $u \in T_{q} \tilde{M}$ is called asymptotic if there is a non zero vector $\nu \in N_{p} M_{\text {sing }}^{k}$ (for $N=3$ ) or $\nu \in E_{p}($ for $N=4)$ such that $I I_{\nu}(u, v)=\langle I I(u, v), \nu\rangle=0$ for all $v \in$ $T_{q} \tilde{M}$. Moreover, in such case, we say that $\nu$ is a binormal direction.

For the case $N=4$, the normal directions $\nu \in N_{p} M_{\text {sing }}^{2}$ which are not in the plane $E_{p}$, but also satisfy the condition $I I_{\nu}(u, v)=\langle I I(u, v), \nu\rangle=$ 0 , are called degenerate directions. The subset of degenerate directions in $N_{p} M_{\text {sing }}^{2}$ is a cone and the binormal directions are those in the intersection of this cone with $E_{p}$.

\section{Normal sections}

Consider $M_{\text {reg }}^{3} \subset \mathbb{R}^{3+k}, k \geq 1$, a regular 3 -manifold (resp. $M_{\text {sing }}^{3} \subset \mathbb{R}^{5}$ a singular corank 13 -manifold). Let $u$ be a tangent direction in $T_{p} M_{\text {reg }}^{3}$ (resp. $T_{p} M_{\text {sing }}^{3}$ ) and $\{u=0\}$ the hyperplane in $\mathbb{R}^{3+k}$ (resp. $\mathbb{R}^{5}$ ) orthogonal to $u$. The normal section of $M_{\text {reg }}^{3}$ along $u$ is a regular surface $M_{\text {reg }}^{2}=M_{\text {reg }}^{3} \cap\{u=0\}$ contained in $\mathbb{R}^{3+k} \cap\{u=0\} \cong \mathbb{R}^{2+k}$ (resp. the normal section $M_{\text {sing }}^{2}$ along $u$ is a singular corank 1 surface $M_{\text {sing }}^{2}=M_{\text {sing }}^{3} \cap\{u=0\}$ contained in $\mathbb{R}^{5} \cap\{u=0\} \cong \mathbb{R}^{4}$ ). 
There is a relation between the curvature locus of $M_{\mathrm{reg}}^{3} \subset \mathbb{R}^{3+k}$ at $p$ and the curvature ellipse of $M_{\mathrm{reg}}^{2}$ at $p$ (resp. the curvature locus of $M_{\text {sing }}^{3} \subset \mathbb{R}^{5}$ at $p$ and the curvature parabola of $M_{\text {sing }}^{2}$ at the same point).

Theorem 3.1. Let $M_{\mathrm{reg}}^{3} \subset \mathbb{R}^{3+k}, k \geq 1$, be a regular 3 -manifold and $p \in M_{\mathrm{reg}}^{3}$. The curvature locus of $M_{\mathrm{reg}}^{3}$ at $p$ is generated by the union of the curvature ellipses at $p$ of the regular surfaces in $\mathbb{R}^{2+k}$ given by the normal sections along the tangent directions of $M_{\mathrm{reg}}^{3}$.

Proof: A normal section induces a hyperplane section of $T_{p} M_{\mathrm{reg}}^{3}$. This hyperplane section intersects the 2 -sphere of unit tangent vectors in an $\mathbb{S}^{1}$. The second fundamental form restricted to this $\mathbb{S}^{1}$ coincides with the second fundamental form of the surface $M_{\text {reg }}^{2}$ obtained as a normal section. Therefore, the curvature ellipses of the normal sections generate the curvature locus of $M_{\text {reg }}^{3}$ at $p$.

Example 3.2. (i) Let $M_{\text {reg }}^{3} \subset \mathbb{R}^{6}$ be given by $f:\left(\mathbb{R}^{3}, 0\right) \rightarrow\left(\mathbb{R}^{6}, 0\right)$,

$$
f(x, y, z)=\left(x, y, z, \frac{\sqrt{2}}{2} x y, \frac{\sqrt{2}}{2} x z, \frac{\sqrt{2}}{2} y z\right) .
$$

At the origin $p$, its curvature locus is a Roman Steiner surface. Taking coordinates $(X, Y, Z, W, T, S)$ in the target, the normal sections given by $\{X=0\},\{Y=0\}$, and $\{Z=0\}$ are regular surfaces whose curvature ellipses at $p$ are, respectively:

$$
\begin{gathered}
\eta_{X}(\theta)=(0,0, \sqrt{2} \sin \theta \cos \theta), \quad \eta_{Y}(\theta)=(0, \sqrt{2} \sin \theta \cos \theta, 0), \\
\eta_{Z}(\theta)=(\sqrt{2} \sin \theta \cos \theta, 0,0),
\end{gathered}
$$

where $\theta \in[0,2 \pi]$. These curvature ellipses are segments which correspond to the double point curves of the Roman Steiner surface. The normal sections $\{X=Y\},\{X=Z\}$, and $\{Y=Z\}$, after changes of coordinates in the source and rotations in the tangent spaces of the surfaces in $\mathbb{R}^{5}$, yield the following curvature ellipses:

$$
\begin{aligned}
& \eta_{X Y}(\theta)=\left(\frac{\sqrt{2}}{2} \sin ^{2} \theta, \sin \theta \cos \theta, \sin \theta \cos \theta\right), \\
& \eta_{X Z}(\theta)=\left(\sin \theta \cos \theta, \frac{\sqrt{2}}{2} \sin ^{2} \theta, \sin \theta \cos \theta\right), \\
& \eta_{Y Z}(\theta)=\left(\sin \theta \cos \theta, \sin \theta \cos \theta, \frac{\sqrt{2}}{2} \sin ^{2} \theta\right),
\end{aligned}
$$

where $\theta \in[0,2 \pi]$. These three curves are non degenerate ellipses. Figure 5 shows the curvature ellipses on the Roman Steiner surface. It appears Steiner himself already knew how to generate the Roman surface by 
ellipses (see [1]). However, all his ellipses pass through a "pole" whereas all of the ellipses obtained here pass through the triple point.

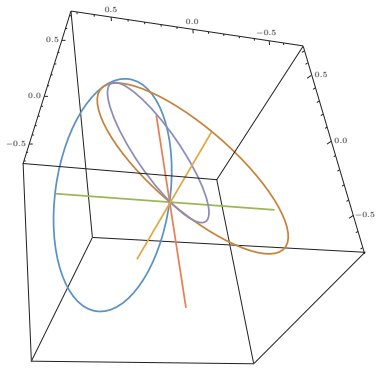

Figure 5. Curvature ellipses on the Roman Steiner surface.

(ii) Let $M_{\text {reg }}^{3} \subset \mathbb{R}^{5}$ be given by $f(x, y, z)=\left(x, y, z, x^{2}+z^{2}, x y\right)$. Taking coordinates $(X, Y, Z, W, T)$ in $\mathbb{R}^{5}$, its curvature locus at the origin $p$ is an elliptic region contained in the normal plane $\{W, T\}$, with center at $(1,0)$ and radius 1 . Table 1 shows some curvature ellipses of regular surfaces given by normal sections. Here, $\theta \in[0,2 \pi]$. Figure 6 shows the curves in Table 1.

\begin{tabular}{cll}
\hline Normal section & Parametrisation of the curvature ellipse & Type \\
\hline$\{X=0\}$ & $\left(2 \sin ^{2} \theta, 0\right)$ & segment \\
$\{Y=0\}$ & $(2,0)$ & point \\
$\{Z=0\}$ & $\left(2 \sin ^{2} \theta, 2 \sin \theta \cos \theta\right)$ & circle \\
$\{X=Z\}$ & $\left(2 \sin ^{2} \theta, \frac{2}{\sqrt{2}} \sin \theta \cos \theta\right)$ & ellipse \\
$\{Y=Z\}$ & $\left(2 \sin ^{2} \theta+\cos ^{2} \theta, \frac{2}{\sqrt{2}} \sin \theta \cos \theta\right)$ & ellipse \\
$\{X=Y\}$ & $\left(\sin ^{2} \theta+2 \cos ^{2} \theta, \sin ^{2} \theta\right)$ & segment \\
\hline
\end{tabular}

TABLE 1.

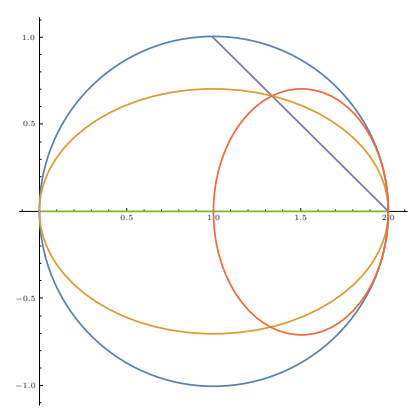

Figure 6. Curvature ellipses on the elliptic region. 
Although it was known that the Roman Steiner surface could be generated by ellipses, geometrically speaking this is not so obvious for the cross-cap, the Steiner type 5, or the cross-cup surfaces.

Theorem 3.3. Let $M_{\text {sing }}^{3} \subset \mathbb{R}^{5}$ be a singular corank 13 -manifold. The curvature locus of $M_{\text {sing }}^{3}$ at $p$ is generated by the union of the curvature parabolas at $p$ of the singular surfaces in $\mathbb{R}^{4}$ given by the normal sections along the tangent directions of $M_{\text {sing }}^{3}$.

Proof: The proof follows as in the regular case. The only different consideration is that $T_{p} M_{\text {sing }}^{3}$ is a plane and if $w \in T_{p} M_{\text {sing }}^{3}$ is a non zero vector, then $\left(d g_{q}\right)^{-1}(w) \subset T_{q} \tilde{M}$ is a plane which contains the subset $\operatorname{ker}\left(d g_{q}\right)$, where $g$ is the corank 1 map at $q$ used in the initial construction and $g(q)=p$.

Hence, the subset $C_{q}^{\prime}=\left(d g_{q}\right)^{-1}(w) \cap C_{q}$ is a pair of lines contained in the unit cylinder $C_{q}$ and such that $\eta_{q}\left(C_{q}^{\prime}\right)$ is the curvature parabola at $p$ of the singular surface contained in the 4-space given by the normal section $\{w=0\}$. Figure 7 shows the previous construction.

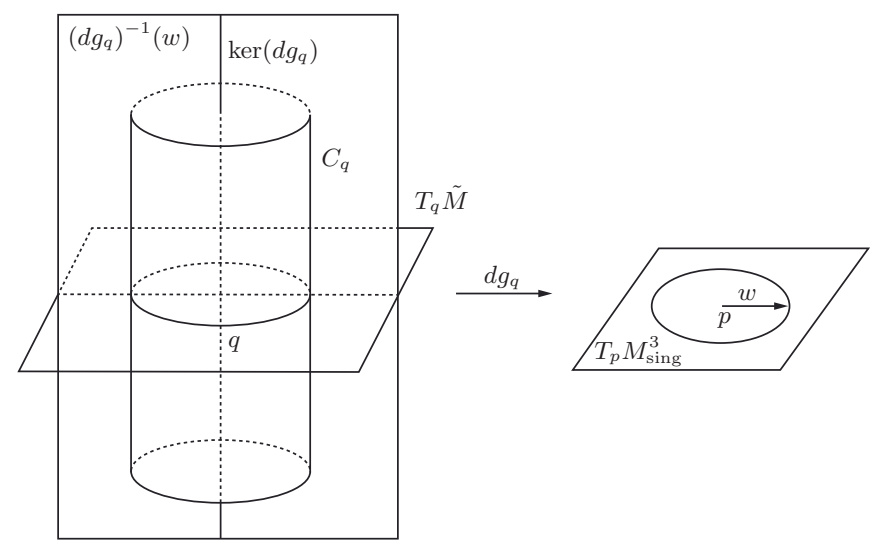

FiguRE 7. Theorem 3.3 .

Example 3.4. Let $M_{\text {sing }}^{3} \subset \mathbb{R}^{5}$ be the singular 3-manifold at the origin $p$ locally given by $f(x, y, z)=\left(x, y, x^{2}-2 y z, y^{2}-2 x z, z^{2}-2 x y\right)$, whose curvature locus $\Delta_{c v}$ at $p$ is

$$
\left\{\left(2 \alpha^{2}-4 \beta \gamma, 2 \beta^{2}-4 \alpha \gamma, 2 \gamma^{2}-4 \alpha \beta\right) \in N_{p} M_{\text {sing }}^{3}: \alpha^{2}+\beta^{2}=1\right\} ;
$$



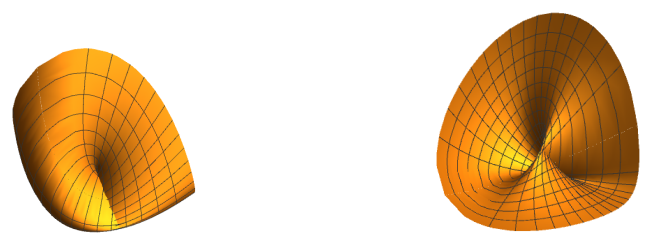

Figure 8. Side and top views of $\Delta_{c v}$.

see Figure 8. The normal section given by $\{Y=0\}$ is parametrised by $\tilde{f}(x, z)=\left(x, x^{2},-2 x z, z^{2}\right)$ and its curvature parabola is a non degenerate parabola, $\tilde{\eta}(z)=\left(2,-4 z, 2 z^{2}\right)$. Taking the normal section $\{X+a Y=0\}$, $a \in \mathbb{R}$, after changes of coordinates at the source and isometries at the target, we obtain the singular surface given by

$\left(0, y, \frac{a^{2} \sqrt{a^{2}+1} y^{2}-2\left(a^{2}+1\right) y z}{\left(a^{2}+1\right)^{3 / 2}}, \frac{\sqrt{a^{2}+1} y^{2}+2 a\left(a^{2}+1\right) y z}{\left(a^{2}+1\right)^{3 / 2}}, \frac{\left(a^{2}+1\right) z^{2}+2 a y^{2}}{a^{2}+1}\right)$,

and $\Delta_{c v}$ is parametrised by

$$
\begin{aligned}
& \eta_{a}(z) \\
= & \left(\frac{2 a^{2} \sqrt{a^{2}+1}-4\left(a^{2}+1\right) z}{\left(a^{2}+1\right)^{3 / 2}}, \frac{2 \sqrt{a^{2}+1}+4 a\left(a^{2}+1\right) z}{\left(a^{2}+1\right)^{3 / 2}}, \frac{4 a+2\left(a^{2}+1\right) z^{2}}{a^{2}+1}\right),
\end{aligned}
$$

a non degenerate parabola for $a \in \mathbb{R}$. Figure 9 shows some of the curvature parabolas in the curvature locus.

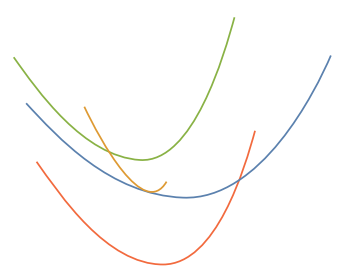

Figure 9. Curvature parabolas.

\section{Relating second order geometry through projections and normal sections}

When projecting a regular $k$-manifold in $\mathbb{R}^{N}$ along a tangent direction we obtain a singular $k$-manifold in $\mathbb{R}^{N-1}$. It is natural to expect certain relations between the curvature loci in each case. For example, in [3] we showed the relation between the curvature ellipse of $M_{\mathrm{reg}}^{2} \subset \mathbb{R}^{4}$ and the curvature parabola of the projection $M_{\text {sing }}^{2} \subset \mathbb{R}^{3}$ and obtained some relations between their second order geometry. It is also known that in the 
previous case, the tangent direction is asymptotic if and only if the singularity of the projection is worse than a cross-cap $([\mathbf{9}, \mathbf{1 9}])$. Similarly, for projections from $M_{\text {reg }}^{2} \subset \mathbb{R}^{5}$ to $M_{\text {sing }}^{2} \subset \mathbb{R}^{4}$, the direction is asymptotic if and only if the singularity is worse than an $I_{1}$-singularity $([\mathbf{2 5}])$.

In the previous section, geometrical relations between manifolds are obtained by normal sections. The following result justifies why relations through projections and normal sections are possible.

Theorem 4.1. Let $M_{\mathrm{reg}}^{3} \subset \mathbb{R}^{6}$ be a regular 3 -manifold, $v \in T_{p} M_{\mathrm{reg}}^{3}$, and $\pi_{v}$ the projection along the direction $v$. Consider a normal section $\Sigma$ such that it contains $v$. Let $i_{1}, i_{2}$ be the immersions of the normal sections in $\mathbb{R}^{6}$ and $\mathbb{R}^{5}$, respectively. Let $v^{\prime}=i_{1_{*}}^{-1}(v) \in T_{i_{1}^{-1}(p)} M_{\text {reg }}^{2}$. We have a commutative diagram

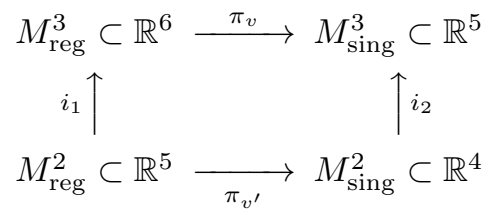

where $M_{\text {reg }}^{2}=M_{\text {reg }}^{3} \cap \Sigma$ and $M_{\text {sing }}^{3}, M_{\text {sing }}^{2}$ are the corresponding singular projections, which induces a commutative diagram amongst the curvature loci of the four manifolds.

Proof: For simplicity, we take $M_{\text {reg }}^{3}$ given in Monge form by

$$
(x, y, z) \mapsto\left(x, y, z, f_{1}(x, y, z), f_{2}(x, y, z), f_{3}(x, y, z)\right),
$$

and fix the direction of projection $v=(0,0,1)$ and the normal section $\Sigma=$ $\{Y=0\}$. In this setting $v^{\prime}=(0,1)$. Consider $(X, Y, Z, W, T, S)$ to be the coordinates of $\mathbb{R}^{6}$. Then $i_{1}$ and $i_{2}$ are given by $i_{1}(X, Z, W, T, S)=$ $(X, 0, Z, W, T, S)$ and $i_{2}(X, W, T, S)=(X, 0, W, T, S)$. We have that $M_{\text {reg }}^{2}$ is given by $\left(x, z, f_{1}(x, 0, z), f_{2}(x, 0, z), f_{3}(x, 0, z)\right)$ and clearly $\pi_{v} \circ$ $i_{1}\left(M_{\text {reg }}^{2}\right)=i_{2} \circ \pi_{v^{\prime}}\left(M_{\text {reg }}^{2}\right)$.

Now, the curvature locus of $M_{\mathrm{reg}}^{3}$ is the image by $I I$ of the unit tangent vectors in $T_{p} M_{\mathrm{reg}}^{3}$. We can parametrise the sphere $\mathbb{S}^{2}$ of unit tangent vectors in spherical coordinates by $(\theta, \phi)$, where $\theta \in[0,2 \pi]$ is the azimuth (i.e. the angle from the $X$-axis in a plane of constant height) and $\phi \in[0, \pi]$ is the polar angle (i.e. the angle from the $Z$-axis). When projecting along the tangent direction $v=(0,0,1)$ we obtain a singular 3 -manifold. The unit tangent vectors in $T_{\pi_{v}(p)} \tilde{M}_{\text {sing }}^{3}$ form a cylinder $C$ which is obtained by blowing up the north and south poles of $\mathbb{S}^{2}$. There is a natural map from $\mathbb{S}^{2}$ to $C$ which takes the spherical coordinates $(\sin \phi \cos \theta, \sin \phi \sin \theta, \cos \phi)$ to the cylindrical coordinates $\left(\cos \theta, \sin \theta, \frac{\cos \phi}{\sin \phi}\right)$ by dividing each component by $\sin \phi$ (i.e. it maps 
the point of intersection with $\mathbb{S}^{2}$ of a ray from the origin to the point of intersection with $C$, the north and south poles go to infinity). This map induces a relation between the parametrisations of the curvature locus of $M_{\mathrm{reg}}^{3}$ and $M_{\mathrm{sing}}^{3}$. In fact, since the loci are the image of $I I$ and the coefficients of these second fundamental forms are the same in the regular and singular cases, the fact of $I I$ being a quadratic homogeneous map means that if $\eta_{e}(\theta, \phi)$ is the parametrisation of the curvature locus of $M_{\mathrm{reg}}^{3}$, then

$$
\eta_{p}(\theta, \phi)=\frac{1}{\sin ^{2} \phi} \eta_{e}(\theta, \phi)
$$

is the parametrisation of the curvature locus of $M_{\text {sing }}^{3}$.

On the other hand, the section $\{Y=0\}$ induces a section in $T_{p} M_{\text {reg }}^{3}$. In spherical coordinates, this gives the section $\{\theta=0\}$ of $\mathbb{S}^{2}$. So, by Theorem 3.1 the curvature ellipse of $M_{\text {reg }}^{2}$ is given by $\eta_{e}(0, \phi)$. Similarly, by Theorem 3.3 the curvature parabola of $M_{\text {sing }}^{2}$ is given by $\eta_{p}(0, \phi)$.

It remains to see that to pass from the curvature ellipse to the curvature parabola we must divide each component of the parametrisation by $\sin ^{2} \phi$. This follows from the geometrical interpretation of $\cot \phi=$ $\frac{\cos \phi}{\sin \phi}$, which again shows that we must divide the components $(\sin \phi, \cos \phi)$ of $\mathbb{S}^{1}$ by $\sin \phi$ to get the components of the unit tangent vectors in $T_{p} \tilde{M}_{\text {sing }}^{2}$, and the fact that the second fundamental form is a homogeneous quadratic map.

Example 4.2. Consider $M_{\text {reg }}^{3}$ given by $f(x, y, z)=\left(x, y, z, x^{2}+\frac{1}{2} z^{2}, x z\right.$, $y z)$. The projection along the tangent vector $(0,0,1)$ is $M_{\text {sing }}^{3}$, given by $\left(x, y, x^{2}+\frac{1}{2} z^{2}, x z, y z\right)$, and the normal section $\{Y=0\}$ gives the regular surface $M_{\text {reg }}^{2}$ by $\left(x, z, x^{2}+\frac{1}{2} z^{2}, x z, 0\right)$. The normal section of $M_{\text {sing }}^{3}$, which coincides with the projection of $M_{\text {reg }}^{2}$ along the tangent vector $(0,1)$, is given by $\left(x, x^{2}+\frac{1}{2} z^{2}, x z, 0\right)$. The curvature locus of $M_{\text {reg }}^{3}$ is a Roman Steiner surface parametrised by

$$
\eta_{e}(\theta, \phi)=\left(1+\sin ^{2} \phi \cos (2 \theta), \cos \theta \sin (2 \phi), \sin \theta \sin (2 \phi)\right),
$$

and the curvature locus of $M_{\text {sing }}^{3}$ is a surface given by

$$
\begin{aligned}
\eta_{p}(\theta, \phi) & =\frac{1}{\sin ^{2} \phi}\left(1+\sin ^{2} \phi \cos (2 \theta), \cos \theta \sin (2 \phi), \sin \theta \sin (2 \phi)\right) \\
& =\left(2 a^{2}+c^{2}, 2 a c, 2 b c\right),
\end{aligned}
$$

where $a=\cos \theta, b=\sin \theta$, and $c=\frac{\cos \phi}{\sin \phi}$, so $a^{2}+b^{2}=1$. The normal section of $M_{\text {sing }}^{3}$ is given by $\{\theta=0\}=\{a=1, b=0\}$, so we get a curvature parabola $\left(2+c^{2}, 2 c, 0\right)$. 
On the other hand, the curvature ellipse of $M_{\text {reg }}^{2}$ is parametrised by $\left(1+\sin ^{2} \phi, \sin (2 \phi), 0\right)$. Dividing by $\sin ^{2} \phi$ and changing $\frac{\cos \phi}{\sin \phi}=c$ we again obtain the curvature parabola $\left(2+c^{2}, 2 c, 0\right)$.

Remark 4.3. Since the curvature locus contains all the second order geometry of the manifold, the previous theorem proves that the second order geometries of $M_{\text {reg }}^{3} \subset \mathbb{R}^{6}, M_{\text {reg }}^{2} \subset \mathbb{R}^{5}, M_{\text {sing }}^{3} \subset \mathbb{R}^{5}$, and $M_{\text {sing }}^{2} \subset \mathbb{R}^{4}$ are all related with one another.

\section{Asymptotic directions of singular 3 -manifolds in $\mathbb{R}^{5}$}

Let $M$ be a corank 1 singular 3-manifold in $\mathbb{R}^{5}, p \in M$, and take $M$ as the image of a smooth map $g: \tilde{M} \rightarrow \mathbb{R}^{5}$, where $\tilde{M}$ is a regular 3-manifold and $q \in \tilde{M}$ is a corank 1 point of $g$ such that $g(q)=p$.

Definition 5.1. A direction $u \in T_{q} \tilde{M}$ is called an asymptotic direction of $M$ at $p$ if there is a non zero vector $\nu \in N_{p} M$ such that

$$
I I_{\nu}(u, v)=\langle I I(u, v), \nu\rangle=0 \quad \forall v \in T_{q} \tilde{M} .
$$

Moreover, in this case, we say that $\nu$ is a binormal direction.

In Theorem 2.2 of [12] several equivalent notions are given to define asymptotic directions in the regular case. We will prove a similar result for the singular case, but before proving this we need the following definition due to Dreibelbis (adapted to the singular case here).

Definition 5.2. Let $\left\{e_{1}, e_{2}, e_{3}\right\}$ be a basis for $T_{q} \tilde{M}$ and $\left\{n_{1}, n_{2}, n_{3}\right\}$ be a basis for $N_{p} M$. For any vector $u \in T_{q} \tilde{M}$, define $A(u)$ as the $3 \times 3$ matrix with $A(u)_{i j}=I I_{n_{i}}\left(e_{j}, u\right)=\left\langle I I\left(e_{j}, u\right), n_{i}\right\rangle$.

Given a unit direction $\nu \in \mathbb{S}^{4}$, the height function of $M \subset \mathbb{R}^{5}$ along $\nu$ is given by $h_{\nu}: M \rightarrow \mathbb{R}, h_{\nu}(p)=\langle p, \nu\rangle$.

Proposition 5.3. Given $u \in T_{q} \tilde{M}$, the following are equivalent:

(1) $u$ is an asymptotic direction.

(2) $\operatorname{det} A(u)=0$.

(3) There exists $\nu \in N_{p} M$ such that the height function $h_{\nu}$ has a degenerate singularity and $u \in$ ker Hess $h_{\nu}$.

Proof: Let $u=\alpha \partial_{x}+\beta \partial_{y}+\gamma \partial_{z}, v=\bar{\alpha} \partial_{x}+\bar{\beta} \partial_{y}+\bar{\gamma} \partial_{z}$, and $\nu=\nu_{1} n_{1}+$ $\nu_{2} n_{2}+\nu_{3} n_{3}$. If $u \in T_{q} \tilde{M}$ is an asymptotic direction we have

$$
\begin{aligned}
I I_{\nu}(u, v)= & \bar{\alpha}\left[\sum_{i=1}^{3} \nu_{i}\left(\alpha l_{n_{i}}+\beta m_{n_{i}}+\gamma q_{n_{i}}\right)\right]+\bar{\beta}\left[\sum_{i=1}^{3} \nu_{i}\left(\alpha m_{n_{i}}+\beta n_{n_{i}}+\gamma r_{n_{i}}\right)\right] \\
& +\bar{\gamma}\left[\sum_{i=1}^{3} \nu_{i}\left(\alpha p_{n_{i}}+\beta q_{n_{i}}+\gamma r_{n_{i}}\right)\right]=0 .
\end{aligned}
$$


This must be satisfied for all $v=(\bar{\alpha}, \bar{\beta}, \bar{\gamma}) \in T_{q} \tilde{M}$, so we get a homogeneous system of three equations in variables $\nu_{1}, \nu_{2}, \nu_{3}$ whose coefficient matrix is precisely $A(u)$. This proves the first equivalence.

Rewriting this system we can see that $u \in \operatorname{kerHess} h_{\nu}$ and that $\operatorname{det}$ Hess $h_{\nu}$ must be 0 , which proves the second equivalence.

Let $M_{\text {sing }}^{3} \subset \mathbb{R}^{5}$ be the projection under a tangent direction of $M_{\text {reg }}^{3} \subset$ $\mathbb{R}^{6}$ and $p^{\prime} \in M_{\text {reg }}^{3}$ the point which is projected to $p$. Since the coefficients of the second fundamental form are the same for both manifolds, the height functions are the same. Therefore, by (3) in Proposition 5.3 and its regular counterpart (Theorem 2.2 in [12]), the binormal directions are the same and $u \in T_{p^{\prime}} M_{\mathrm{reg}}^{3}$ is asymptotic if and only if $u \in T_{q} \tilde{M}$ is asymptotic.

On the other hand, by direct calculation of the coefficients of the first fundamental form for the singular projection in terms of the coefficients of the first fundamental form for the regular manifold, we can see that the direction of projection $u$ becomes the null tangent direction of the singular projection (this explains the concept of infinite asymptotic directions appearing in $[\mathbf{1 6}]$ or $[\mathbf{5}])$.

By the above discussion we get the following:

Theorem 5.4. Let $M_{\text {sing }}^{3} \subset \mathbb{R}^{5}$ be the projection under a tangent direction of $M_{\mathrm{reg}}^{3} \subset \mathbb{R}^{6}$. The direction of projection is asymptotic for $M_{\mathrm{reg}}^{3} \subset$ $\mathbb{R}^{6}$ if and only if the null tangent direction is asymptotic for $M_{\text {sing }}^{3} \subset \mathbb{R}^{5}$.

Definition 5.5. When the null tangent direction $u \in T_{p} M_{\text {sing }}^{n}$ is asymptotic we call it infinite asymptotic direction and denote it by $u_{\infty}$.

The idea of an infinite asymptotic direction is as follows. Any singular manifold can be seen as the projection of a regular manifold along a tangent direction. A singular manifold with an infinite asymptotic direction necessarily comes from a regular manifold projected along an asymptotic direction (which has become the infinite asymptotic direction).

In the case of $M_{\text {reg }}^{2} \subset \mathbb{R}^{4}$ projected to $M_{\text {sing }}^{2} \subset \mathbb{R}^{3}$ the following are equivalent:

(i) The direction of projection is an asymptotic direction.

(ii) $M_{\text {sing }}^{2} \subset \mathbb{R}^{3}$ has a singularity worse than a cross-cap.

(iii) The curvature parabola $\Delta_{p}$ of $M_{\text {sing }}^{2} \subset \mathbb{R}^{3}$ is degenerate.

(The equivalence between (i) and (ii) can be found in $[\mathbf{1 9}, \mathbf{9}]$ and the equivalence between (ii) and (iii) is shown in [16].) Therefore, by Theorem 5.4 adapted to these dimensions (the proof is the same), for $M_{\text {sing }}^{2} \subset \mathbb{R}^{3}$ there is an infinite asymptotic direction only if $\Delta_{p}$ is degenerate. 
For $M_{\text {reg }}^{3} \subset \mathbb{R}^{6}$ projected to $M_{\text {sing }}^{3} \subset \mathbb{R}^{5}$, an analogous result to the equivalence between (i) and (ii) can be proved using results from [6]. However, the equivalence between (i) and (iii) for 3-manifolds is not true in general (see [6] for a partial result):

Example 5.6. Let $M_{\text {reg }}^{3} \subset \mathbb{R}^{6}$ be given by $\left(x, y, z, x^{2}+z^{2}, x y+x z, y^{2}\right)$. The direction $u=(0,0,1) \in$ ker Hess $h_{\nu}$ for the binormal direction $\nu=(0,0,1)$, so $u$ is an asymptotic direction. Projection along $u$ yields $\left(x, y, x^{2}+\right.$ $\left.z^{2}, x y+x z, y^{2}\right)$ and the curvature locus is given by

$$
\left(\cos ^{2} \theta+\frac{\cos ^{2} \phi}{\sin ^{2} \phi}, \cos \theta \sin \theta+\cos \theta \frac{\cos \phi}{\sin \phi}, \sin ^{2} \theta\right)
$$

which is not contained in a plane, i.e. it is not a degenerate curvature locus.

The previous example shows that in some cases a non-degenerate curvature locus has an infinite asymptotic direction, so we must define the image of $u_{\infty}$ and its tangent space for some $M_{\text {sing }}^{3} \subset \mathbb{R}^{5}$ with nondegenerate curvature locus.

Definition 5.7. Let $\eta(\theta, \phi)$ denote the parametrisation of the curvature locus $\Delta_{c v}$ of $M_{\text {sing }}^{3}$. For each topological type of the curvature locus we must define $\eta\left(u_{\infty}\right)$ :

(i) If $\Delta_{c v}$ is a point $r$, then $\eta\left(u_{\infty}\right)=r$ and $\frac{\partial \eta}{\partial \theta}\left(u_{\infty}\right)=\frac{\partial \eta}{\partial \phi}\left(u_{\infty}\right)=0$.

(ii) If $\Delta_{c v}$ is a line or a half-line, then $\eta\left(u_{\infty}\right)=\frac{\partial \eta}{\partial \theta}\left(u_{\infty}\right)=\frac{\partial \eta}{\partial \phi}\left(u_{\infty}\right)=$ $\frac{\eta^{\prime}(t)}{\left|\eta^{\prime}(t)\right|}$ for any $t$ such that $\eta^{\prime}(t) \neq 0$, where $t$ is the parameter of the line.

(iii) If $\Delta_{c v}$ is a planar region or a plane, then $\eta\left(u_{\infty}\right)=\frac{\partial \eta}{\partial \theta}\left(u_{\infty}\right)=\frac{\partial \eta}{\partial \theta}(v)$ for any $v$ such that $\eta(v)$ does not lie in the boundary of $\Delta_{c v}$ and $\frac{\partial \eta}{\partial \phi}\left(u_{\infty}\right)=\left(\frac{\partial \eta}{\partial \theta}(v)\right)^{\perp}$.

(iv) If $\Delta_{c v}$ is non-degenerate such that $u_{\infty}$ is an asymptotic direction of $M_{\text {reg }}^{3}$, then $\eta\left(u_{\infty}\right)=\frac{\partial \eta}{\partial \theta}\left(u_{\infty}\right)=\frac{\partial \eta}{\partial \phi}\left(u_{\infty}\right)=\lim _{\phi \rightarrow 0} \frac{\eta(\theta, \phi)}{|\eta(\theta, \phi)|}$.

We can now add an equivalence to Proposition 5.3:

Theorem 5.8. Let $u \in C_{q} \cup\left\{u_{\infty}\right\}$. Then $u$ is asymptotic if and only if

(4) The vector $\eta(u)$ is tangent to $\eta\left(C_{q} \cup\left\{u_{\infty}\right\}\right)$ at $\eta(u)$ or $\Delta_{c v}=\eta\left(C_{q}\right)$ is singular at $u$.

Proof: Suppose first that $u$ is not the null tangent direction. In this case we proceed as in $[\mathbf{1 2}]$. Let $C_{q}$ be parametrised by $(\theta, \phi)$ in cylindrical coordinates and let $u=u(\theta, \phi)$. Then $\frac{\partial \eta(u)}{\partial \theta}=I I(u, u)_{\theta}=2 I I\left(u, u_{\theta}\right)$ 
and $\frac{\partial \eta(u)}{\partial \phi}=I I(u, u)_{\phi}=2 I I\left(u, u_{\phi}\right)$. Since $\left\{u, u_{\theta}, u_{\phi}\right\}$ are linearly independent, having the tangency or a singularity means that $\{I I(u, u)$, $\left.I I(u, u)_{\theta}, I I(u, u)_{\phi}\right\}=\left\{I I(u, u), 2 I I\left(u, u_{\theta}\right), 2 I I\left(u, u_{\phi}\right)\right\}$ is linearly dependent, and this happens if and only if there exists a unit tangent vector $w \in C_{q}$ such that $I I(u, w)=0$. Consider now $g=I I(u, \cdot): T_{q} \tilde{M} \rightarrow$ $N_{p} M$. Since $w \in \operatorname{ker} g$, the image of $g$ is contained in a plane in $N_{p} M$ and so, what we have is equivalent to the fact that there exists $\nu \in N_{p} M$ such that $I I_{\nu}(u, v)=0$ for all $v \in T_{q} \tilde{M}$, i.e. $u$ is asymptotic.

For $u_{\infty}$ the tangency occurs by construction of $\frac{\partial \eta}{\partial \theta}\left(u_{\infty}\right)$ and $\frac{\partial \eta}{\partial \phi}\left(u_{\infty}\right)$.

Theorem 5.9. Let $M_{\text {sing }}^{2} \subset \mathbb{R}^{4}$ be a normal section given by $M_{\text {sing }}^{3}$, $p \in M_{\text {sing }}^{3}$, and suppose that $\operatorname{Aff}_{i_{2}^{-1}(p)}=E_{i_{2}^{-1}(p)}$. Then $u \in T_{q} \tilde{M}$ is an asymptotic direction of $M_{\mathrm{sing}}^{3}$ if and only if $\left(i_{2_{*}}\right)^{-1}(u)$ is an asymptotic direction of $M_{\text {sing }}^{2}$.

Proof: Lemma 4.10 in [5] is the equivalent result to Theorem 5.8 for singular surfaces in $\mathbb{R}^{4}$. Taking a normal section is taking a hyperplane $U$ in $T_{q} \tilde{M}$. This induces an intersection of $\Delta_{c v}$ with the plane $I I(U)$. A direction $u \in T_{q} \tilde{M}$ is asymptotic if $\eta(u)$ is tangent to $\eta\left(C_{q} \cup\left\{u_{\infty}\right\}\right)$ or $\eta\left(C_{q}\right)$ is singular at $u$. The curvature parabola of $M_{\text {sing }}^{2} \subset \mathbb{R}^{4}$ is given by $\Delta_{c v} \cap$ $I I(U)$ and here $\eta(u)$ is tangent to $\Delta_{c v} \cap I I(U)$ if and only if $I I(U)$ is a plane that passes through the origin, i.e. $\operatorname{Aff}_{i_{2}^{-1}(p)}=E_{i_{2}^{-1}(p)}$. Hence, $\eta_{\left.\right|_{U \cap C_{q}}}\left(\left(i_{2_{*}}\right)^{-1}(u)\right) \in E_{i_{2}^{-1}(p)}$ and is also parallel to $\eta_{\left.\right|_{U \cap C_{q}} ^{\prime}}^{\prime}\left(\left(i_{2_{*}}\right)^{-1}(u)\right)$, that is, $\left(i_{2_{*}}\right)^{-1}(u)$ is an asymptotic direction of the normal section.

Example 5.10. Consider the singular 3-manifold given by $\left(x, y, x^{2}-\right.$ $\left.2 y z, y^{2}-2 x z, z^{2}-2 x y\right)$. Its curvature locus is given by

$$
\left(2 \cos ^{2} \theta-4 \sin \theta \frac{\cos \phi}{\sin \phi}, 2 \sin ^{2} \theta-4 \cos \theta \frac{\cos \phi}{\sin \phi}, 2 \frac{\cos ^{2} \phi}{\sin ^{2} \phi}-4 \cos \theta \sin \theta\right) \text {. }
$$

We have that $(0,1,-1)$ is an asymptotic direction associated to the binormal direction $(-1,1,1)$. Consider now the normal section given by $\{X=$ $0\}$ and parametrised by $\left(y,-2 y z, y^{2}, z^{2}\right)$. The curvature parabola is given by $\left(-4 y, 2,2 y^{2}\right)$. Here $(-1,1,1)$ is a degenerate direction, but it is not binormal since it is not in $E_{p}$, and therefore $(1,-1)$ is not an asymptotic direction of the singular surface.

Remark 5.11. Proposition 5.3 and Theorem 5.8 can be generalised to other dimensions. The setting in this section works well for $M_{\mathrm{reg}}^{k} \subset \mathbb{R}^{2 k}$ projected to $M_{\text {sing }}^{k} \subset \mathbb{R}^{2 k-1}$ and taking normal sections of the singular manifold to get $M_{\text {sing }}^{k-1} \subset \mathbb{R}^{2(k-1)}$. 


\section{References}

[1] F. ApÉRY, "Models of the Real Projective Plane", Computer graphics of Steiner and Boy surfaces, With a preface by Egbert Brieskorn, Friedr. Vieweg \& Sohn, Braunschweig, 1987. DOI : 10.1007/978-3-322-89569-1.

[2] P. Benedini Riul, Geometria diferencial de segunda ordem de variedades singulares em espaços euclidianos, Ph.D. thesis, Universidade de São Paulo (2019).

[3] P. Benedini Riul and R. Oset Sinha, A relation between the curvature ellipse and the curvature parabola, Adv. Geom. 19(3) (2019), 389-399. DOI: 10.1515/ advgeom-2019-0002.

[4] P. Benedini Riul and R. Oset Sinha, The flat geometry of the $I_{1}$ singularity: $(x, y) \mapsto\left(x, x y, y^{2}, y^{3}\right)$, J. Singul. 21 (2020), 1-14. DOI: 10.5427/jsing. 2020. 21a.

[5] P. Benedini Riul, M. A. S. Ruas, and R. Oset Sinha, The geometry of corank 1 surfaces in $\mathbb{R}^{4}$, Q. J. Math. 70(3) (2019), 767-795. DOI: 10.1093/ qmath/hay064.

[6] P. Benedini Riul, M. A. S. Ruas, and A. de J. Sacramento, Singular 3-manifolds in $\mathbb{R}^{5}$, Preprint (2019). arXiv:1911.00360.

[7] R. R. Binotto, S. I. R. Costa, and M. C. Romero Fuster, The curvature Veronese of a 3-manifold immersed in Euclidean space, in: "Real and Complex Singularities", Contemp. Math. 675, Amer. Math. Soc., Providence, RI, 2016, pp. 25-44. DOI : 10.1090/conm/675.

[8] R. R. Binotto, S. I. R. Costa, and M. C. Romero Fuster, Geometry of 3-manifolds in Euclidean space, in: "Theory of Singularities of Smooth Mappings and Around it", RIMS Kôkyûroku Bessatsu B55, Res. Inst. Math. Sci. (RIMS), Kyoto, 2016, pp. 1-15.

[9] J. W. Bruce and A. C. Nogueira, Surfaces in $\mathbf{R}^{4}$ and duality, Quart. J. Math. Oxford Ser. (2) 49(4) (1998), 433-443. DOI : 10.1093/qmathj/49.4.433.

[10] J. W. Bruce and F. Tari, Families of surfaces in $\mathbb{R}^{4}$, Proc. Edinb. Math. Soc. (2) 45(1) (2002), 181-203. DOI : 10.1017/S0013091500000213.

[11] S. I. R. Costa, S. M. Moraes, and M. C. Romero-Fuster, Geometric contacts of surfaces immersed in $\mathbb{R}^{n}, n \geq 5$, Differential Geom. Appl. 27(3) (2009), 442-454. DOI : $10.1016 / \mathrm{j}$.difgeo.2008.10.020.

[12] D. Dreibelbis, Self-conjugate vectors of immersed 3-manifolds in $\mathbb{R}^{6}$, Topology Appl. 159(2) (2012), 450-456. DOI : 10.1016/j.topol.2011.09.019.

[13] R. A. Garcia, D. K. H. Mochida, M. C. Romero Fuster, and M. A. S. Ruas, Inflection points and topology of surfaces in 4-space, Trans. Amer. Math. Soc. 352(7) (2000), 3029-3043. DOI : 10.1090/S0002-9947-00-02404-1.

[14] S. Izumiya, M. C. Romero Fuster, M. A. S. Ruas, and F. Tari, "Differential Geometry from a Singularity Theory Viewpoint", World Scientific Publishing Co. Pte. Ltd., Hackensack, NJ, 2016.

[15] J. A. LitTle, On singularities of submanifolds of higher dimensional Euclidean spaces, Ann. Mat. Pura Appl. (4) 83 (1969), 261-335. DOI : 10.1007/BF02411172.

[16] L. F. Martins and J. J. Nuño-Ballesteros, Contact properties of surfaces in $\mathbb{R}^{3}$ with corank 1 singularities, Tohoku Math. J. (2) 67(1) (2015), 105-124. DOI : $10.2748 / \mathrm{tmj} / 1429549581$.

[17] D. K. H. Mochida, M. C. Romero Fuster, And M. A. S. Ruas, The geometry of surfaces in 4-space from a contact viewpoint, Geom. Dedicata 54(3) (1995), 323-332. DOI : 10.1007/BF01265348. 
[18] D. K. H. Mochida, M. C. Romero-Fuster, and M. A. S. Ruas, Osculating hyperplanes and asymptotic directions of codimension two submanifolds of Euclidean spaces, Geom. Dedicata 77(3) (1999), 305-315. DOI : 10.1023/A: 1005145104603.

[19] D. M. Q. Mond, Classification of certain singularities and applications to differential geometry, Ph. D. thesis, University of Liverpool (1982).

[20] S. M. Moraes and M. C. Romero-Fuster, Convexity and semiumbilicity for surfaces in $\mathbb{R}^{5}$, in: Differential Geometry, Valencia 2001", World Sci. Publ., River Edge, NJ, 2002, pp. 222-234. DOI : 10.1142/4922.

[21] J. J. Nuño Ballesteros, M. C. Romero Fuster, and F. Sánchez-Bringas, Curvature locus and principal configurations of submanifolds of Euclidean space, Rev. Mat. Iberoam. 33(2) (2017), 449-468. DOI : 10.4171/RMI/944.

[22] J. J. Nuño-Ballesteros and F. Tari, Surfaces in $\mathbb{R}^{4}$ and their projections to 3-spaces, Proc. Roy. Soc. Edinburgh Sect. A 137(6) (2007), 1313-1328. DOI : 10. 1017/S0308210506000758.

[23] R. Oset Sinha and F. Tari, Projections of surfaces in $\mathbb{R}^{4}$ to $\mathbb{R}^{3}$ and the geometry of their singular images, Rev. Mat. Iberoam. 31(1) (2015), 33-50. DOI : 10.4171/ RMI/825.

[24] M. C. Romero-Fuster, Semiumbilics and geometrical dynamics on surfaces in 4-spaces, in: "Real and Complex Singularities", Contemp. Math. 354, Amer. Math. Soc., Providence, RI, 2004, pp. 259-276. DOI : 10.1090/conm/354/06484.

[25] M. C Romero-Fuster, M. A. S. Ruas, and F. Tari, Asymptotic curves on surfaces in $\mathbb{R}^{5}$, Commun. Contemp. Math. 10(3) (2008), 309-335. DOI : 10.1142/ S0219199708002806.

[26] K. Saji, M. Umehara, and K. Yamada, The geometry of fronts, Ann. of Math. (2) 169(2) (2009), 491-529. DOI : 10.4007/annals.2009.169.491.

P. Benedini Riul

Departamento de Matemática, Universidade Federal de São Carlos, Caixa Postal 676, São Carlos, SP 13560-905, Brazil

E-mail address: pedro.benedini.riul@gmail.com

R. Oset Sinha

Departament de Matemàtiques, Universitat de València, Campus de Burjassot, 46100

Burjassot, Spain

E-mail address: raul.oset@uv.es

Primera versió rebuda el 4 de desembre de 2019, darrera versió rebuda el 8 de juliol de 2020 . 